\title{
Etiological Aspects of the Dilatation of the Upper Urinary Tract at University Hospital of Gabriel Toure
}

\author{
Amadou Kassogué ${ }^{*}$, Mamadou Tidiani Coulibaly², Zanafon Ouattara², Adama Yaflé Diarra², \\ Aly Tembely ${ }^{3}$
}

\author{
${ }^{1}$ Department of Urology, University Hospital of Pr Bocar Sidy Sall at Kati, Mali \\ ${ }^{2}$ Department of Urology, University Hospital of Gabriel Toure at Bamako, Mali \\ ${ }^{3}$ Department of Urology, University Hospital of Point G at Bamako, Mali \\ Email: *kassogueamadou@hotmail.fr
}

How to cite this paper: Kassogué, A., Coulibaly, M.T., Ouattara, Z., Diarra, A.Y. and Tembely, A. (2018) Etiological Aspects of the Dilatation of the Upper Urinary Tract at University Hospital of Gabriel Toure. Open Journal of Urology, 8, 199-205.

https://doi.org/10.4236/oju.2018.87022

Received: May 2, 2018

Accepted: July 10, 2018

Published: July 13, 2018

Copyright $\odot 2018$ by authors and Scientific Research Publishing Inc. This work is licensed under the Creative Commons Attribution International License (CC BY 4.0).

http://creativecommons.org/licenses/by/4.0/

(C) (i) Open Access

\begin{abstract}
Introduction: The dilatation of the upper urinary tract, signs in the vast majority of cases the presence of a ureteral obstacle. The etiologies are diverse. Mali is a country where urinary schistosomiasis is endemic. Repeated infestation may result in long-term ureteropelastic dilatation secondary to stenotic sequelae. The objective is to study the causes of dilatations of the upper urinary tract. Materials and Methods: This is a prospective study of 12 months, from October 2010 to September 2011, performed in the urology department of Gabriel Toure University Hospital in Bamako. The parameters studied were: age, sex, reason for consultation, medical history, urinary analysis, etiological diagnosis, management, evaluation of renal failure. Result: In 12 months, we collected 50 cases of dilatations of the upper urinary tract representing $0.66 \%$ of all consultations. The average age of our patients was 35 years old. The sex ratio was 2.13 in favor of men. A history of urinary schistosomiasis was found in 25 patients or $50 \%$. Low back pain was the most common reason for consultation, $76 \%$. The renal and bladder ultrasonography performed in all 50 patients in our series found bilateral dilatation in $68 \%$ of patients and unilateral dilation in $32 \%$ of cases. Urinary tract infection with Escherichia coli was found in 53\% of cases. Ureterovesical reimplantation was performed in $40 \%$ of cases. Conclusion: The dilatation of the upper urinary tract, consequence of an anatomical or functional obstruction, constitutes a rather frequent pathology and constituted $10 \%$ of the surgical acts of our service. Etiologies are diverse: congenital and acquired. Open surgery gives good results, but the introduction of innovative minimally invasive surgical technique is necessary.
\end{abstract}




\section{Keywords}

Etiological, Dilatation of the Upper Urinary Tract, Treatment

\section{Introduction}

Uretero-pyelocalyceal dilatation (UPCD), in the vast majority of cases, indicates the presence of a ureteral obstacle [1]. The etiologies are diverse. This dilatation is discovered as part of a review of renal failure or low back pain. The pelvic and abdominal X-ray/Ultrasound or the abdominopelvic computed tomography scan (CT scan) without first-line injection is urgently needed to confirm the etiological diagnosis [2]. As Mali is part of the endemic bilharzia zone, repeated infestation can lead to long-term uretero-pyelocalyceal dilatation secondary to stenotic sequelae. Their evolutions without treatment result in the alteration of the renal function. Clinical signs are not specific. These uretero-pyelocalyceal dilatations lay a threat to the patient who can progress to acute, chronic or terminal renal failure.

Stenosis of the ureterovesical junction dues to the sequelae of specific urinary tract infections (urinary bilharziasis and urinary tuberculosis) and renal lithiasis are responsible for dilation of the upper urinary tract in our department.

Vamadevan, S., et al. [1], reported that in pediatrics, dilatation of the upper urinary tract is associated with mal formative of the urogenital system, in particular, the ureteropelvic junction obstruction, the vesico ureteral reflux, the mega-ureter, the ureterocele, and the posterior urethralvalve [1]. While for the adult, the majority of the causes of dilatations of the upper urinary tract are obstructive and multiple causes. Ureteral stones are the main cause of intra luminal obstruction, especially in men between 30 and 60 years old.

In older men, dilatation of upper urinary tract may be the result of bladder emptying disorders, in cases of benign or malignant prostatic hypertrophy that may lead to vesico-ureteric reflux [1] [3].

The objective is to study the etiological of dilatations of the upper urinary tract in our department.

\section{Materials and Methods}

Our study was carried out in the urology department of Gabriel Toure University Hospital in Bamako. This is a prospective study of 12 months from October 2010 to September 2011. The study included patients of all ages, of all sex from Bamako and the interior of the country. They were either sent by the doctors of different health facilities, or came to consult themselves at the department.

About the selection criteria, it included all patients admitted and operated for uretero-pyelocalyceal dilatations or hydronephrosis and whose diagnosis was made by clinical and para-clinical means. 
Information about interrogative, general and urological physical examination, biological examinations, medical imaging examinations are collected either directly or on the patient's hospitalization file and have been analyzed statistically. Data collection and analysis focused on aspects, such as surgical management, the first three months of postoperative follow-up, as well as the results of the pathology examinations.

The parameters studied were age, sex, reason for consultation, medical history, Urinary analysis, etiological diagnosis, management, evaluation of renal function.

\section{Result}

In 12 months, we collected 50 cases (Table 1) of dilatations of the upper urinary tract representing $0.66 \%$ of all consultations.

The average age of our patients was 35 years old. The sex ratio was 2.13 in favor of men. A history of urinary schistosomiasis was found in 25 patients or $50 \%$. Low back pain was the most common reason for consultation, $76 \%$. The kidney and bladder ultrasonography performed in all 50 patients in our series found bilateral dilatation in $68 \%$ of patients and unilateral dilation in $32 \%$ of cases. Urinary tract infection with Escherichia coli was found in 53\% of cases (Table 2).

Stenosis of the ureterovesical junction was the most frequent, with $20 \%$ or $40 \%$ followed by lithiasis of the upper urinary system (kidney and ureter) $16 \%$ or $30 \%$ (Table 3). Of the 20 cases of ureterovesical junction stenosis, we performed an ureterovesical reimplantation in all patients (Table 4). Two cases of nephrectomy were performed for coralliform lithiasis with destruction of the renal parenchyma. Congenital malformations such as: ureteropelvic junction obstruction, posterior urethral valve have also been found to be a possible cause of dilatation of the upper excretory tract.

The dilatation of the excretory tract, a consequence of an anatomic or functional obstruction, is a fairly frequent pathology (10.31\% of the Service's surgical procedures). It mainly affects the young adult male. We had 3 cases of

Table 1. Distribution of patients according by age.

\begin{tabular}{ccc}
\hline Age & Effectifs & Percentage (\%) \\
$0-10$ & 2 & 4 \\
$11-20$ & 4 & 8 \\
$21-30$ & 11 & 22 \\
$31-40$ & 8 & 16 \\
$41-50$ & 5 & 10 \\
$51-60$ & 9 & 18 \\
61 and more & 11 & 22 \\
Total & 50 & 100 \\
\hline
\end{tabular}


Table 2. Distribution of patients according to the result of the urinary analysis.

\begin{tabular}{ccc}
\hline Urinary analysis & Effectifs & Percentage (\%) \\
\hline Escherichia coli & 20 & 53 \\
Proteus mirabilis & 6 & 16 \\
Candidat albicans & 5 & 13 \\
Klebssiela pneumoniae & 2 & 5 \\
Trichomonas vaginalis & 2 & 5 \\
Streptococus aureus & 2 & 5 \\
Enterobacter & 1 & 3 \\
Total & 38 & 100 \\
\hline
\end{tabular}

Urine analysis identified a urinary tract infection in 38 cases. E. coli was the responsible germ in 20 cases or $53 \%$.

Table 3. Distribution of patients according to the etiological diagnosis.

\begin{tabular}{ccc}
\hline Preoperative Diagnosis & Effectifs & Percentage (\%) \\
\hline Stenosis of the ureterovesical junction & 20 & 40 \\
Kidney stone & 12 & 24 \\
BPH & 7 & 14 \\
Ureteral stone & 4 & 8 \\
Bladder cancer & 3 & 6 \\
Posterior urethral valve & 2 & 4 \\
Uretero-pelvic junction obstruction & 2 & 4 \\
Total & 50 & 100 \\
\hline
\end{tabular}

Stenosis of the ureterovesical junction obstruction was the most common with $40 \%$ of renal lithiasis cases.

Table 4. Distribution of patients according by surgery.

\begin{tabular}{ccc}
\hline Surgery & Effectifs & Percentage (\%) \\
\hline Ureterovesical reimplantation & 20 & 40 \\
Nephrolithotomy & 7 & 14 \\
Prostatectomy & 7 & 14 \\
Ureterolithotomy & 4 & 8 \\
Pyelolithotomy & 3 & 6 \\
Autres & 3 & 6 \\
Nephrectomy & 2 & 4 \\
Pyeloplasty & 2 & 4 \\
Valvedestruction & 2 & 4 \\
Total & 50 & 100 \\
\hline
\end{tabular}

Ureterovesical reimplantation was the most commonly practiced technique with $40 \%$ of cases.

bladder cancer. The evolution was favorable in 47 patients, i.e. $94 \% .3$ cases of complications were recorded: 1 case of cutaneous vesico fistula, 1 case of death and 1 case of parietal suppuration. Preoperatively, renal function was impaired 
in 19 patients. Of the 19 patients, control of serum creatinine three months after surgery was normal in 9 patients.

\section{Discussion}

Dilatations of the upper urinary tract are frequent reasons for consultation in urology. They can manifest as back pain, renal colic, discovered during an assessment of renal failure, or accidental during an examination requested for other things. Urinary schistosomiasis is endemic in Mali. It can progress silently, either spontaneously healed after treatment but where fibrosclereuses leaving scars and calcifications in the bladder and the intramural portion of the ureter, causing adolescence to adulthood where pathologies obstructive or tumor.

The age groups between $21-30$ and 61 years and over were the most affected with $22 \%$ each. We found a male predominance, 34 men out of 16 women with a sex ratio of 2.13 in favor of men. 25 patients or $50 \%$ of patients had a history of urinary schistosomiasis. The urinary analysis identified a urinary tract infection in 38 cases. E. coli was the responsible germ in 20 cases or 53\%. Renal function was impaired in 19 cases, i.e. 38\% of patients. Medical imaging is unavoidable composed of: ultrasound, pelvic and abdominal X-ray, Intravenous urography (IVU), CT scan. Renal and bladder ultrasonography is used as a first-line examination in our patients. On the ultrasound examination dilatation was bilateral in 34 cases or $68 \%$ of cases.

Intravenous urography: Is the basic examination that allows affirming the diagnosis. It also makes it possible to evaluate the degree of uretero-pyelocalyceal dilatation. Of the 38 patients who performed IVU, we had uretero hydronephrosis in 27 cases (71\%) and hydronephrosis in 11 cases (29\%). Cystoscopy was performed in 8 of our patients, among which we found 3 cases of bladder cancer. Stenosis of the ureterovesical junction was the most common with $40 \%$ of renal lithiasis cases. The etiologies of dilatations of the upper urinary tract are very diverse.

Uro-genital bilharziosis, responsible for stenosis of the lower ureter remains the dominant etiology in our series with $40 \%$ of cases. Renal lithiasis associated with hydronephrosis or ureteral lithiasis as a cause of uretero-pyelocalyceal dilatation was found in $32 \%$ of cases. We had 3 cases of bladder cancer in our series, i.e. $6 \%$ of the sample; all three patients came from a highly endemic environment with a history of terminal hematuria.

Vamadevan, S. et al. [1], reports that the incidence and causes of upper urinary tract dilatation vary by age and sex and that in children this dilatation is associated with malformative uropathy: ureteropelvic junction, vesicoureteral reflux (VUR), mega ureter, ureterocele, posterior urethral valve. In adults, ureteral stones are the main cause of intraluminal obstruction, especially in men between the ages of 30 and 60 , and benign or malignant prostatic hypertrophy in older men.

Evolution of impaired renal function: the control of serum creatinine three 
months after surgery, 40 cases or $95 \%$ of patients normalized their renal function. We consider that the 2 remaining cases constitute a chronic renal failure. Coulibalily, N., in Ivory Coast [4], reports that obstructions of the upper urinary tract are frequently responsible for fatal renal failure in $29.23 \%$ of cases and that the etiologies are pelvic cancers (uterus cervix, bladder, prostate).

The importance of a rapid diagnosis before sepsis develops, exposing the patient to a life-threatening risk was emphasized by Guerrot, D., et al. [5]. The criteria for a urological opinion in the face of dilatation are: the septic state, the acute renal failure, the notion of a single kidney, the bilateral dilatation, the renal colic not responding to a well-conducted analgesic treatment [1]. Urinary drainage from the upper urinary tract by placement of a double J ureteral catheter or nephrostomy is indicated. Hydronephrosis with signs of severity is a vital emergency and requires urinary drainage [1]. Roy, C., et al. [6] reports that urinary obstruction occurs in three forms: acute, chronic and intermittent. Biological examinations (serum creatinine, urinary analysis); Morphological examinations (ultrasound, IVU, CT scan, voiding cystourethrography (VCUG) and renal scintigraphy) can be a valuable contribution to the monitoring and evaluation of the result of the management of these obstructive uropathies.

Despite this etiological heterogeneity of our series, the therapeutic aim sought remains the same, namely to restore the permeability of the urinary excretory pathways and to avoid complications. Ureterovesical reimplantation accounted for $40 \%$ of the acts. The insufficiency of our technical platform has limited us to use only surgery "open air" to ensure the care of 47 patients. The other 3 patients underwent transuretral resection of prostate (TURP) in another department.

For Descote, J.L. [7], ureteropelvic junction is the most frequent cause of hydronephrosis, the diagnosis of which is often evoked on antenatal ultrasound examinations and secondarily confirmed on postnatal examinations. The diagnosis is mostly postnatal in the context. The development of laparoscopic or robot assisted techniques makes it possible to hope for shorter hospital stays while maintaining the good results of this intervention [8].

Fritschi, U. et al. [9], reports that in the presence of a stone of $6 \mathrm{~mm}$ to $2 \mathrm{~cm}$ or in the event of failure of conservative treatment, the indications of ureteral stones, the treatment of choice for the stone located below the iliac crest is semi-rigid ureteroscopy, and extracorporeal shockwave lithotripsy (ESWL) for localized stones above.

Open surgery remains effective, but in the face of changes in the standard of living of the population and the increase of expectations regarding the results of the interventions, the introduction of innovative minimally invasive surgical technique is more than necessary. The management of obstructive uropathies in this last decade has been increasingly marked by a growing interest in endoscopic surgery. This is because of the advantages it offers in terms of improving the aesthetic result, the reduction of operating morbidity and the rapid recovery 
of daily activities. The insufficiency of our technical platform has led us to use only surgery "open air" to ensure the care of 47 patients. These different aspects constitute the limit of our study.

\section{Conclusion}

The dilatation of the excretory tract, consequence of an anatomic or functional obstruction, constitutes a rather frequent pathology and $10 \%$ of the surgical acts of our department. It mainly affects the young adult male. Etiologies are diverse: congenital and acquired. Uro-genital bilharziasis with its stenosing sequelae is the predominant antecedent affecting both kidneys in the majority of cases. Open surgery gives good results, but the introduction of innovative minimally invasive surgical technique is necessary.

\section{Conflicts of Interest}

No conflict interest.

\section{References}

[1] Vamadevan, S., Klein, J. and Iselin, C.E. (2015) How to Menage a Pyelocalyceal Dilatation? Revue Médicale Suisse, 11, 2293-2297.

[2] Grenier, N. and Taourel, P. (2004) Imaging of Acute Urinary Obstruction: NonEnhanced CT or KUB and US? Journal de Radiologie, 85, 186-194. https://doi.org/10.1016/S0221-0363(04)97568-3

[3] Klahr, S. (2000) Obstructive Nephropathy. Internal Medicine, 39, 355-361. https://doi.org/10.2169/internalmedicine.39.355

[4] Coulibalily, N., Dje, K., Yao, H., Boka, M.D., Kouakou, A., Akassimadou, N., et al. (2016) Obstruction of the Upper Urinary Tract: Circumstances of Diagnosis and Methods of Drainage. Revue Africaine d Urologie et d Andrologie, 1, 233-236.

[5] Guerrot, D. and Tamion, F. (2013) Obstructive Acute Renal Failure: The Intensivist's Viewpoint. Progrès en Urologie, 23, 19-22. https://doi.org/10.1016/j.fpurol.2012.10.001

[6] Roy, C. and Buy, X. (2003) Urinary Obstruction: Different Radio-Clinical Types. Journal de Radiologie, 84, 109-119.

[7] Descote, J.L. (2013) Management of Adult Uretero-Pelvic Junction Obstruction. Progrès en Urologie, 23, 1172-1176.

[8] Francesca, M.M., Bahler, C.D., Scheinder, E.B. and Sundaram, C.P. (2013) Emerging Trends in Robotic Pyeloplasty for the Management of Ureteropelvic Junction Obstruction in Adults. Journal of Urology, 189, 1352-1357. https://doi.org/10.1016/j.fpurol.2012.10.001

[9] Fritschi, U., Kabongo, O.M., Tawadros, T., Jisclinski, P. and Valerio, M. (2014) Multimodal Surgical Management of Stones in the Upper Urinary Tract. Revue Médicale Suisse, 10, 2316-2321. 